\title{
Spatial heterogeneity of intracardiac 4D relative pressure fields during diastole
}

\author{
Jonatan Eriksson ${ }^{1,2^{*}}$, Ann Bolger ${ }^{4,1}$, Tino Ebbers ${ }^{1,2}$, Carl Johan Carlhall ${ }^{2,3}$ \\ From 16th Annual SCMR Scientific Sessions \\ San Francisco, CA, USA. 31 January - 3 February 2013
}

\section{Background}

Blood flow within the cardiovascular system is driven by pressure differences, where blood accelerates from higher to lower pressure areas. Invasive methods of pressure measurement, which are commonly applied to assessment of diastolic function, may not capture the heterogeneity of regional intracardiac pressure differences. We utilized pressure fields based on time-resolved 3D CMR data to investigate the timing and distribution of intracardiac pressure gradients in the left heart throughout diastole.

\section{Methods}

12 healthy subjects ( 5 female, age $47 \pm 17$ y.o. (mean \pm sd)) underwent MRI examination (1.5T, Philips Achieva) where $4 \mathrm{D}$ velocity and morphological short (SA) and long axis bSSFP data were acquired. Acquisition parameters for the velocity sequence were: TE $3.7 \mathrm{~ms}$, TR $6.3 \mathrm{~ms}$, spatial resolution $3 \times 3 \times 3 \mathrm{~mm} 3$, and $\mathrm{k}$-space segmentation factor 2 . This resulted in a temporal resolution of $50.4 \mathrm{~ms}$. The field-of-view was adjusted to fit each subject.

The left ventricle (LV) and atrium (LA) were segmented at all diastolic time frames using freely available software Segment (http://segment.heiberg.se). The segmentation was used as boundary condition for computation of the $4 \mathrm{D}$ relative pressure field, based on the pressure Poisson equation (Ebbers and Farnebäck, 2009 JMRI). The pressure data were visualized using the commercial software package EnSight (figure 1). The pressure range for the

entire LA and LV volume at each diastolic time frame was calculated and the maximum value identified in each subject. Further, the pressure range in the full segmented LV volume was calculated and related to the pressure range along a line from LA to LV (figure 1), at the time of maximum positive pressure gradient between LA and LV during early filling (TimeOfMaxPressure).

\section{Results}

The maximum pressure range anywhere in the combined LA and LV volume at any time during diastole was $3.2 \pm 1.1 \mathrm{mmHg}$. At TimeOfMaxPressure, the pressure range anywhere in the $L V$ volume was $2.0 \pm 1.0 \mathrm{mmHg}$. At the same time point, the pressure range along the atrium-to-apex line was $1.7 \pm 0.6 \mathrm{mmHg}$ (figure 2), which represented $89 \pm 21 \%$ of the pressure range anywhere in the LV volume.

\section{Conclusions}

This CMR study implies that intracardiac pressure gradients are spatially heterogenous during the diastolic phase. These results emphasize the benefit of pressure assessment from methods that take into account the 3D nature of the intracardiac pressure field. These findings may also impact the interpretation of clinical catheterbased intracardiac pressure measurements.

\section{Funding}

This study was funded by the Swedish heart-lung foundation and the Swedish research council. 


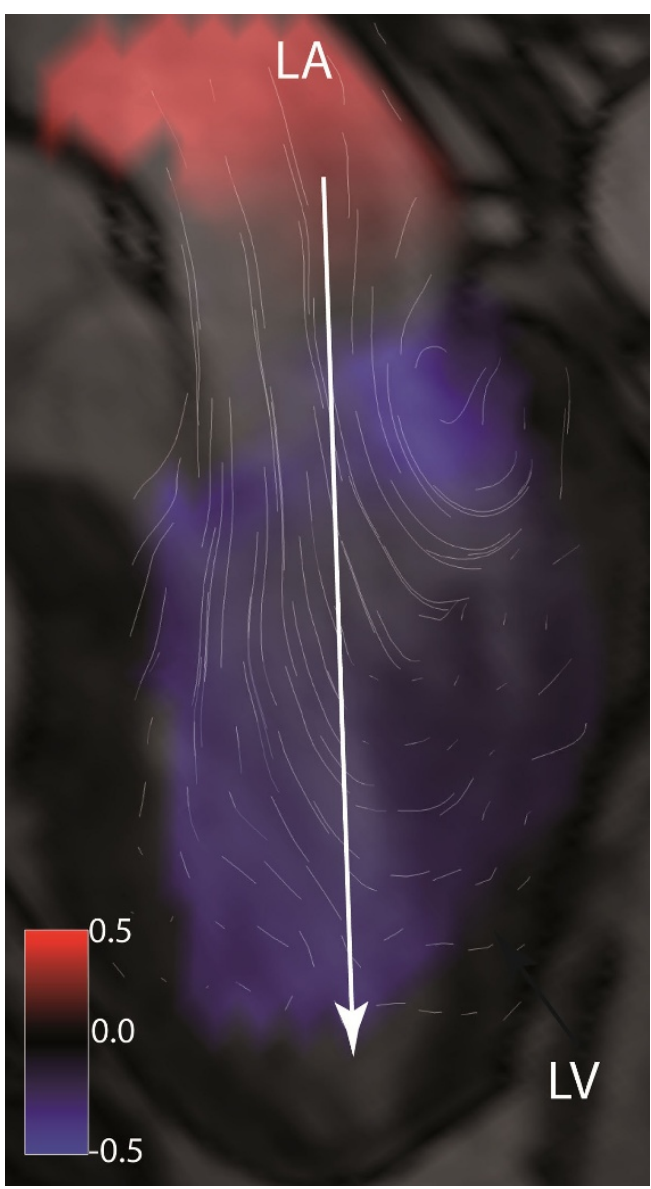

Figure 1 Visualization of relative pressure $(\mathrm{mmHg})$ at Time Of Max Pressure in a healthy 61 y.o. male with a heart rate of $61 \mathrm{bpm}$. A morphological three-chamber image is included for orientation. Certain data were extracted from a line through the 3D data set from left atrium (LA) to left ventricular (LV) apex (arrow).

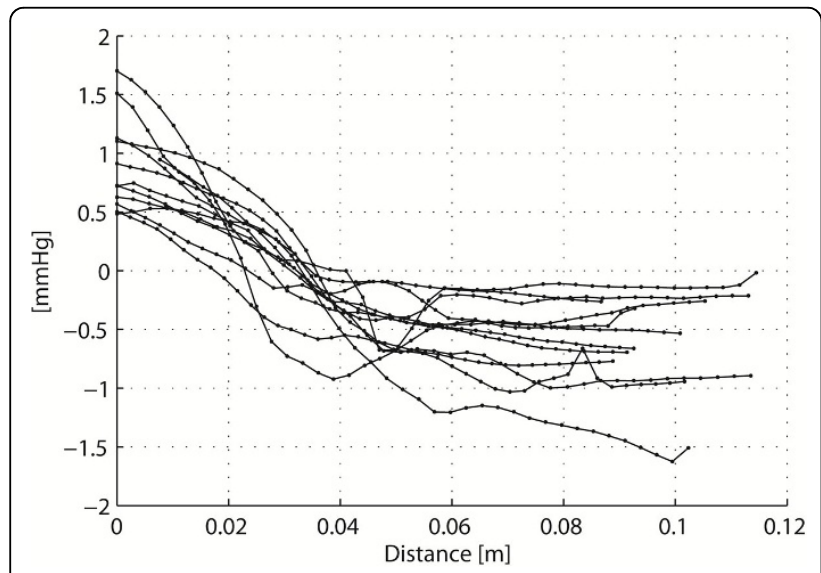

Figure 2 Relative pressure $(\mathrm{mmHg})$ along a line between left atrium (LA) and left ventricular (LV) apex, (figure 1) at TimeOfMaxPressure. Data represent 12 healthy subjects. The $x$-axis show distance in meters from the start of the line in the atrium to the ventricular apex.

\section{Author details}

${ }^{1}$ Department of Medical and Health Sciences, Linköping University, Linköping, Sweden. ${ }^{2}$ Center for Medical Image Science and Visualization (CMIV), Linköping University, Linköping, Sweden. ${ }^{3}$ Department of Clinical Physiology UHL, County Council of Östergötland, Linköping, Sweden. ${ }^{4}$ Department of Medicine, University of California, San Francisco, CA, USA.

Published: 30 January 2013

doi:10.1186/1532-429X-15-S1-M13

Cite this article as: Eriksson et al:: Spatial heterogeneity of intracardiac $4 \mathrm{D}$ relative pressure fields during diastole. Journal of Cardiovascular Magnetic Resonance 2013 15(Suppl 1):M13.

\section{Submit your next manuscript to BioMed Central and take full advantage of:}

- Convenient online submission

- Thorough peer review

- No space constraints or color figure charges

- Immediate publication on acceptance

- Inclusion in PubMed, CAS, Scopus and Google Scholar

- Research which is freely available for redistribution 